\title{
Larvas de trematódeos de Biomphalaria spp. (Gastropoda: Planorbidae) de dois municípios do leste da Amazônia Legal brasileira
}

\section{Larval trematodes in Biomphalaria spp. (Gastropoda: Planorbidae) from two municipalities of eastern Brazilian Legal Amazon}

João Gustavo Mendes Rodrigues' ', Guilherme Silva Miranda', Maria Gabriela Sampaio Lira', Ranielly Araújo Nogueira', Gleycka Cristine Carvalho Gomes ${ }^{1}$, Remy Santos Cutrim², Nêuton Silva-Souza ${ }^{1}$

1 Universidade Estadual do Maranhão, Departamento de Química e Biologia, São Luís, Maranhão, Brasil

2 Universidade Federal do Maranhão, Centro de Ciências da Saúde, Departamento de Biologia, São Luís, Maranhão, Brasil

\begin{abstract}
RESUMO
OBJETIVO: Realizar um levantamento da biodiversidade de larvas de trematódeos oriundas de caramujos Biomphalaria spp. obtidos de criadouros naturais de dois municípios do estado do Maranhão, na porção leste da Amazônia Legal brasileira. MATERIAIS E MÉTODOS: Os gastrópodes foram coletados bimestralmente em três pontos distintos de São Luís e São Bento, de fevereiro de 2015 a janeiro de 2016. Os moluscos obtidos foram identificados por meio de morfologia externa e interna. As cercárias foram fixadas, coradas e identificadas com o auxílio de chaves taxonômicas. RESULTADOS: Foram coletados 2.661 moluscos em São Luís e 1.726 em São Bento, obtendo-se 3,72\% (99/2.661) e 4,87\% (84/1.726) de planorbídeos positivos para larvas de trematódeos, respectivamente, nas cidades estudadas. A helmintofauna foi representada, em São Luís, por Clinostomidae (0,41\%), Diplostomidae (0,56\%), Echinostomatidae (0,90\%), Schistosomatidae $(0,71 \%)$, Spirorchiidae $(0,41 \%)$ e Strigeidae (0,71\%); e, em São Bento, por Echinostomatidae (1,80\%), Schistosomatidae (0,75\%), Spirorchiidae $(1,56 \%)$ e Strigeidae $(0,75 \%)$. Entre as cercárias da família Schistosomatidae de ambos os municípios, relatou-se apenas a espécie Schistosoma mansoni. Em São Luís, as espécies de Biomphalaria apresentaram as seguintes taxas de infecção: 1,95\% (52/2.661) para Biomphalaria straminea e 1,76\% (47/2.661) para Biomphalaria glabrata; em São Bento, essas taxas foram de 4,46\% (77/1.726) para B. glabrata e 0,40\% (7/1.726) para B. straminea. CONCLUSÃO: As cercárias identificadas, com exceção de S. mansoni, foram consideradas primeiros relatos para São Luís, enquanto que, para São Bento, obteve-se o primeiro relato para a família Strigeidae.
\end{abstract}

Palavras-chave: Helmintofauna; Diversidade; Moluscos.

\begin{abstract}
OBJECTIVE: To carry out a survey about larval trematodes in Biomphalaria spp. snail biodiversity, obtained from natural breeding sites of two municipalities in Maranhão State, eastern Brazilian Legal Amazon. MATERIALS AND METHODS: The gastropods were collected bimonthly at three distinct points in São Luís and São Bento, from February 2015 to January 2016. The mollusks obtained were identified by external and internal morphology. The cercariae were fixed, stained and identified using specific taxonomic keys. RESULTS: A total of 2,661 molluscs were collected in São Luís and 1,726 in São Bento; and 3.72\% $(99 / 2,661)$ and $4.87 \%(84 / 1,726)$ of planorbids were positive for larval trematodes, respectively. In São Luís, the helminth fauna identified were Clinostomidae (0.41\%), Diplostomidae (0.56\%), Echinostomatidae (0.90\%), Schistosomatidae (0.71\%), Spirorchiidae (0.41\%), and Strigeidae $(0.71 \%)$ families; and in São Bento were Echinostomatidae (1.80\%), Schistosomatidae (0.75\%), Spirorchiidae (1.56\%), and Strigeidae (0.75\%) ones. Among cercariae in Schistosomatidae family of the both municipalities, only Schistosoma mansoni was reported. In São Luís, Biomphalaria species presented the following infection rates: $1.95 \%(52 / 2,661)$ for Biomphalaria straminea and 1.76\% (47/2,661) for Biomphalaria glabrata. In São Bento, these rates were $4.46 \%(77 / 1,726)$ for $B$. glabrata and $0.40 \%(7 / 1,726)$ for B. straminea. CONCLUSION: The identified cercariae, with the exception of S. mansoni, are considered first reports for São Luís; while for São Bento the first report is for the Strigeidae family.
\end{abstract}

Keywords: Helmintofauna; Diversity; Mollusks.

\author{
Correspondência / Correspondence: \\ João Gustavo Mendes Rodrigues \\ Universidade Estadual do Maranhão \\ Cidade Universitária Paulo VI, s/n. Bairro: Tirirical - C.P. 09 - CEP: 65055-970 - São Luís, Maranhão, Brasil - Tel.: + 55 (98) 98845-1066 \\ E-mail: gustavorodrigues_98@hotmail.com
}




\section{INTRODUÇÃO}

As larvas de trematódeos, também denominadas de cercárias, são organismos de vida livre que emergem de moluscos aquáticos, geralmente em grande quantidade, fazendo parte do zooplâncton. Ao longo da sua evolução, diversas adaptações biológicas foram surgindo para potencializar ao máximo o encontro das cercárias com seus respectivos hospedeiros. Entre essas adaptações estão a grande replicação desses organismos no interior dos moluscos, a liberação sincronizada das cercárias em momentos que favoreçam o encontro com os seus hospedeiros definitivos e o desenvolvimento de respostas a estímulos físicos, químicos e ambientais em associação à presença desses hospedeiros s, $2,3,4^{1}$.

Os ciclos de vida dos trematódeos digenéticos são complexos, havendo duas principais etapas: a reprodução sexuada nos hospedeiros definitivos (em sua maioria vertebrados); e a reprodução assexuada em hospedeiros intermediários (principalmente gastrópodes aquáticos) ${ }^{5}$. Essas larvas são formadas durante a etapa de reprodução assexuada, por meio da formação de rédias e/ou esporocistos, por um processo denominado de poliembrionia ${ }^{2,6}$.

Diversos tipos e formas larvais de trematódeos oriundos de diferentes espécies de moluscos de água doce no Brasil foram descritos e caracterizados inicialmente por diferentes autores $7,8,9,10,11$, principalmente nos estados das Regiões Sul e Sudeste $12,13,14,15,16$. Desses moluscos, destaca-se 0 caramujo do gênero Biomphalaria, pois ele atua como hospedeiro intermediário do Schistosoma mansoni, agente etiológico da esquistossomose mansônica, além de albergar outros tipos de larvas emergentes ${ }^{17}$.

Entretanto, diferentes cercárias, já identificadas atualmente, apresentam importância médica e veterinária, podendo transmitir dois grupos de doenças: as que possuem transmissão através da penetração direta na pele, como a esquistossomose ${ }^{18}$; e as que são transmitidas pelo consumo de água e alimentos crus ou malcozidos contendo esses parasitos, como a clonorquiose, a heterofiose e a paragonimose ${ }^{19,20}$.

Para a região da Amazônia Legal brasileira, poucos estudos foram realizados tentando conhecer a biodiversidade das larvas de trematódeos, a exemplo da pesquisa realizada por Cantanhede et al. ${ }^{21}$. Essa área abrange os estados da Região Norte, além dos estados do Mato Grosso e Maranhão, a oeste do Meridiano 4422; e é detentora de uma imensa variedade biológica de espécies, ecossistemas e recursos energéticos ${ }^{23}$.

$\mathrm{Na}$ porção leste dessa Região, onde o estado do Maranhão está localizado, encontram-se alguns biomas, como os campos alagados da Baixada Ocidental e o Litoral Norte do Estado, que, devido aos seus aspectos sociais, naturais e geomorfológicos, favorecem a manutenção e abundância de moluscos límnicos, o que consequentemente aumenta as possibilidades de se tornarem potenciais hospedeiros de trematódeos digenéticos ${ }^{24}$. Sendo assim, a riqueza taxonômica de larvas de helmintos parasitos, para a região, encontra-se subestimada. Desse modo, objetivou-se realizar o levantamento da biodiversidade de larvas de trematódeos eliminadas pelos caramujos Biomphalaria spp., obtidos de criadouros naturais de duas localidades do Maranhão, na Amazônia Legal, Brasil.

\section{MATERIAIS E MÉTODOS}

presente estudo foi realizado durante o período de fevereiro de 2015 a janeiro de 2016 em dois municípios do estado do Maranhão que pertencem à porção leste da Amazônia Legal brasileira: São Bento e São Luís. O município de São Bento $\left(2^{\circ} 40^{\prime} 00^{\prime \prime} S\right.$

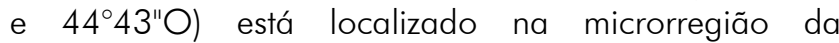
Baixada Ocidental maranhense e mesorregião Norte maranhense, possuindo uma área de 585,3 km² e ambiente caracterizado por apresentar campos naturalmente alagados e clima tropical úmido, destacando-se duas estações climáticas: uma chuvosa, entre janeiro e julho; e outra seca, entre agosto e dezembro ${ }^{25}$. O município de São Luís $\left(2^{\circ} 31^{\prime} \mathrm{LS}\right.$ e $\left.44^{\circ} 18^{\prime} \mathrm{LO}\right)$ está localizado entre as baías de São Marcos e São José de Ribamar, possui área de $822,1 \mathrm{~km}^{2}$ e apresenta clima tropical quente e úmido, com duas estações: a chuvosa (janeiro a junho) e a de estiagem (julho a dezembro) ${ }^{26}$ (Figura 1). Ambos os municípios apresentam bairros periféricos com condições precárias de vida, entre as quais se destacam a falta de saneamento básico, a grande concentração populacional e a presença de coleções hídricas contendo elevado número de caramujos Biomphalaria spp. ${ }^{27}$.

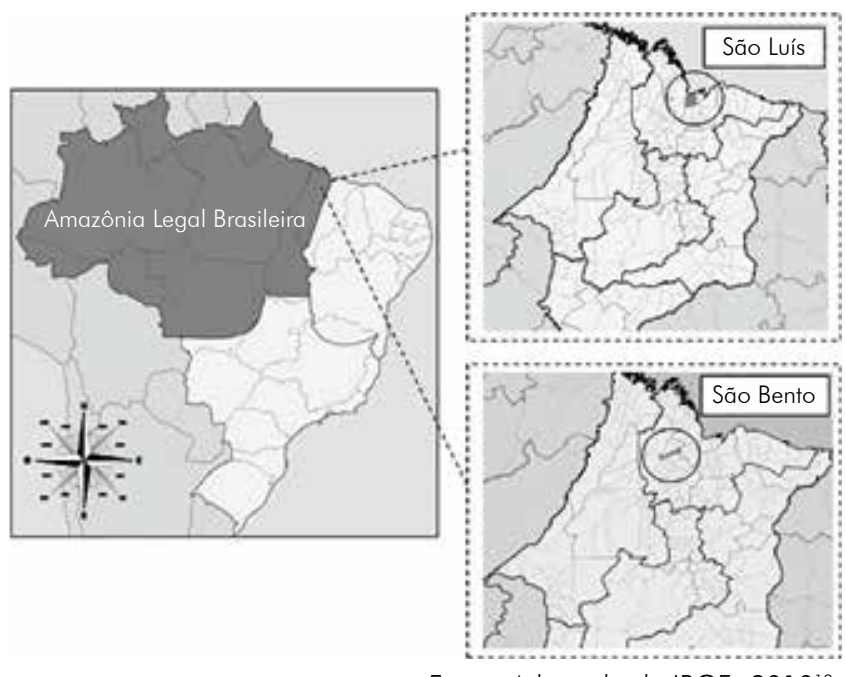

Fonte: Adaptado de IBGE, $2010^{19}$.

Figura 1 - Localização da área de estudo, estado do Maranhão, porção leste da Amazônia Legal brasileira, com destaque para os dois municípios onde foram realizadas as coletas

A coleta dos moluscos foi realizada bimestralmente, durante $30 \mathrm{~min}$, em três pontos (área de 15 a $20 \mathrm{~m}$ ) distintos em cada município, com auxílio de conchas de 
capturas apropriadas e pinças metálicas. A escolha dos pontos de coleta foi feita de modo a se obter maior diversidade de ambientes que poderiam funcionar como criadouros. As coordenadas geográficas foram obtidas por meio de sistema de posicionamento global (global positioning system - GPS). Os moluscos coletados foram armazenados em recipientes plásticos, que foram etiquetados e conduzidos ao Laboratório de Parasitologia Humana da Universidade Estadual do Maranhão (UEMA), onde foram mantidos em aquários de vidro com água desclorada e alimentados adequadamente com folhas de alface, segundo - que preconiza Malek ${ }^{28}$. Aproximadamente 10\% de exemplares vivos foram sacrificados em água a $70{ }^{\circ} \mathrm{C}$, para possibilitar a retirada das partes moles, que foram fixadas em solução de RaillietHenry, que posteriormente foram dissecadas sob estereomicroscópio. Para a identificação das espécies, foram considerados parâmetros conquiliológicos e morfológicos internos ${ }^{29,30}$.

Para a constatação da eliminação de larvas de trematódeos, os moluscos foram individualmente colocados em recipientes de vidro, com cerca de $5 \mathrm{~mL}$ de água desclorada, e expostos à luz e ao calor de três lâmpadas incandescentes (de $60 \mathrm{~W}$ ) durante $4 \mathrm{~h}^{31}$. Para a exposição no escuro, foram deixados dentro de uma caixa de isopor sem tampa, na ausência de luz e calor durante $12 \mathrm{~h}$ e, em seguida, analisados em lupa microscópica. Os moluscos negativos ao teste foram expostos aos mesmos procedimentos semanalmente, durante 30 dias.

As larvas encontradas foram coletadas com auxílio de micropipeta e utilizadas para a confecção de preparações a fresco, que foram coradas com solução de lugol diluída e por corantes vitais ${ }^{31}$, sendo, em seguida, montadas sob lâmina e lamínula. Após isso, foram conduzidas ao microscópio óptico para caracterização e identificação morfológica, segundo chaves de identificação taxonômicas de diferentes autores $5,32,33,34,35,36$. Todas as coletas foram autorizadas pelo Instituto Chico Mendes de Conservação da Biodiversidade, por meio do Sistema de Autorização e Informação em Biodiversidade, de acordo com a licença $n^{\circ} 40025 / 1$ e o registro $n^{\circ} 54354$, válidos para o período de 2015/2016.

\section{RESULTADOS}

Foram coletados moluscos em três pontos de cada município, sendo registradas as seguintes coordenadas geográficas para São Luís: P1 (Bairro

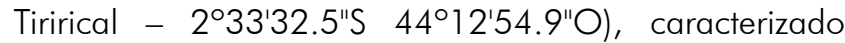
pela presença de alterações antrópicas visíveis, como assoreamento, polvição por dejetos urbanos e retirada de mata ciliar (Figura 2A); P2 (Bairro

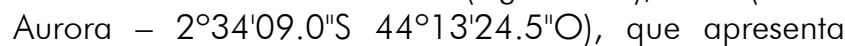
características ambientais mais conservadas, como a presença de mata ciliar do tipo secundária, a ausência de erosão nas margens e assoreamento, além de polvição do tipo urbana pouco evidente (Figura 2B); e P3 (Bairro Sá Viana - 2³2'47.5"S $\left.44^{\circ} 14^{\prime} 06.9^{\prime \prime} \mathrm{O}\right)$, local com um acentuado aglomerado urbano, com a presença de grande quantidade de resíduos domésticos (Figura 2C). Em São Bento: $\mathrm{Pl}$

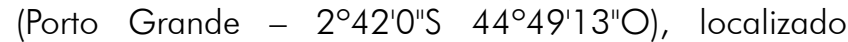
às margens de um dos grandes lagos naturalmente alagáveis do Município, mas onde, devido à proximidade de casas nos arredores, o despejo de esgoto doméstico era evidente, bem como a presença de resíduos urbanos diversos (Figura 3A); P2 (Outra

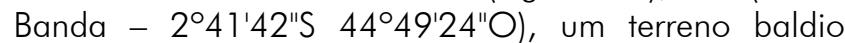
caracterizado por apresentar moderada quantidade de resíduos domésticos, bem como a presença de grande quantidade de habitações próximas (Figura 3B); e P3

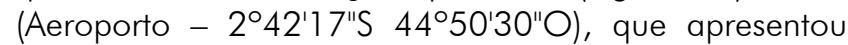
características ambientais visualmente conservadas (Figura 3C).

Em São Luís, coletou-se um total de 2.661 moluscos, que estavam distribuídos nos pontos de coleta da seguinte forma: P1, 432 (16,23\%); P2, 172 (6,46\%); e P3, 2.057 (77,31\%). No município de São Bento, 1.726 moluscos foram capturados e registrados, como se segue: $\mathrm{P1}, 326$ (18,89\%); $\mathrm{P} 2$, $1.214(70,33 \%)$; e P3, 186 (10,78\%). Em ambos os municípios, foram encontrados exemplares de duas espécies de Biomphalaria (Biomphalaria straminea e Biomphalaria glabrata).

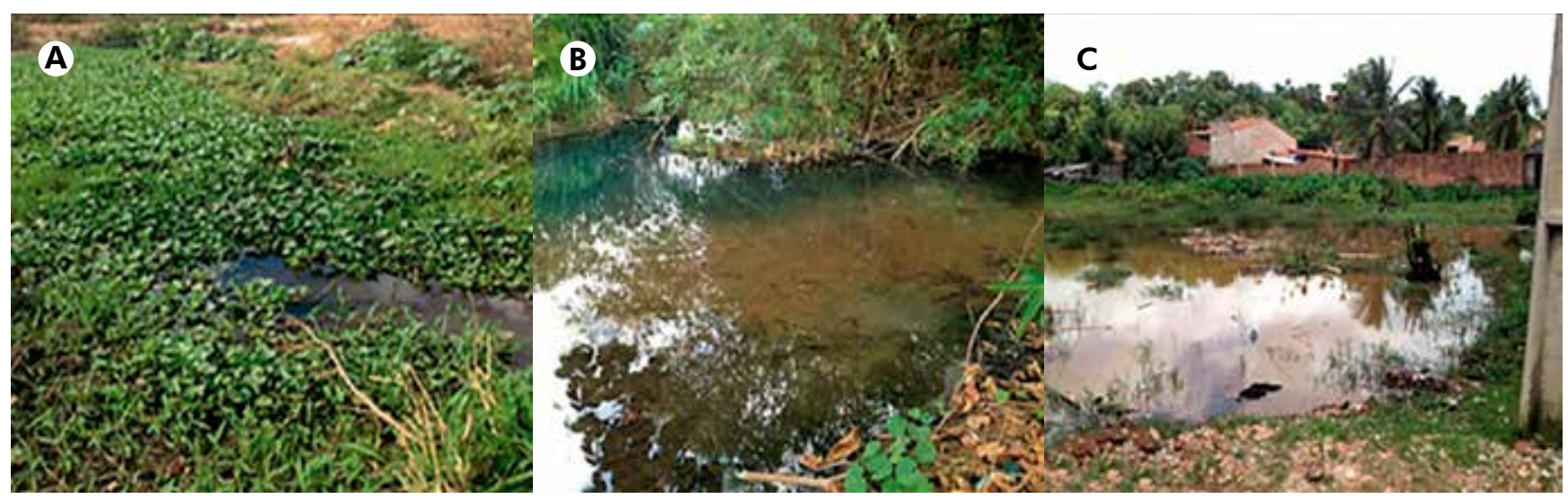

A: Ponto 1 - Bairro Tirirical; B: Ponto 2 - Bairro Aurora; C: Ponto 3 - Bairro Sá Viana.

Figura 2 - Pontos de coleta nos criadouros naturais de Biomphalaria spp. do município de São Luís, estado do Maranhão, Brasil 


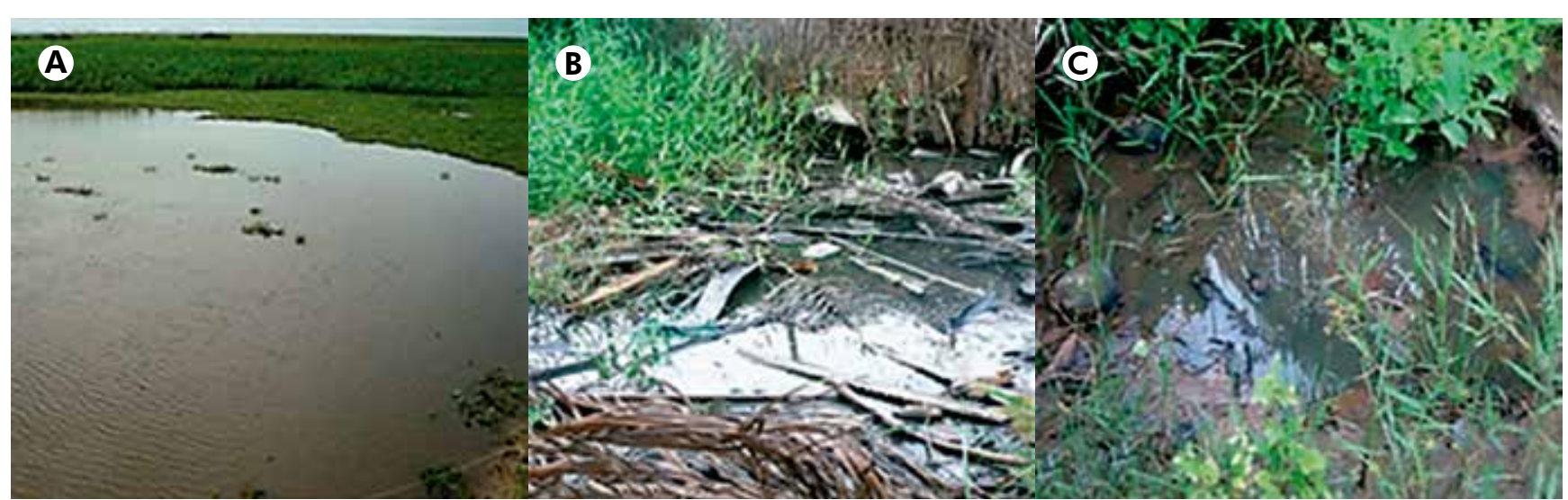

A: Ponto 1 - Bairro Porto Grande; B: Ponto 2 - Bairro Outra Banda; C: Ponto 3 - Bairro Aeroporto.

Figura 3 - Pontos de coleta nos criadouros naturais de Biomphalaria spp. do município de São Bento, estado do Maranhão, Brasil

A positividade desses moluscos para larvas de trematódeos, em São Luís, foi em 99/2.661 (3,72\%) exemplares, com percentuais específicos nos criadouros: $\mathrm{Pl}, 31 / 432$ (7,17\%), P2, 7/172 (4,07\%) e P3, $61 / 2.057$ (2,96\%). Em São Bento, com 84/1.726 $(4,87 \%)$ exemplares, distribuídos em percentuais específicos: P1, 29/326 (8,89\%) e P2, 55/1.214 (4,53\%); não houve moluscos positivos em P3 nessa localidade. Também não foram observados casos de coinfecção entre diferentes larvas de trematódeos nos moluscos dos Municípios.

A helmintofauna da região foi representada por seis famílias de larvas de trematódeos: Clinostomidae, Diplostomidae, Echinostomatidae, Schistosomatidae, Spirorchiidae e Strigeidae. Em São Luís, foram registradas todas essas, mas em São Bento não se obteve 0 registro das famílias Clinostomidae e
Diplostomidae (Figura 4). Ressalta-se ainda que as cercárias da família Schistosomatidae identificadas em São Luís no P3 e em São Bento em P1 e P2 eram todas da espécie $S$. mansoni, o que constitui uma realidade preocupante para a população, por se tratarem de pontos próximos a residências.

As larvas de trematódeos, em São Luís, foram eliminadas com um percentual de 1,95\% (52/2.661) de B. straminea e $1,76 \%(47 / 2.661)$ de B. glabrata. Por sua vez, em São Bento, foram eliminadas com percentual de 4,46\% (77/1.726) de B. glabrata e 0,40\% (7/1.726) de B. straminea (Tabela 1). Todas as formas larvais de trematódeos identificadas neste estudo, com exceção da cercária de S. mansoni, são consideradas primeiros registros para o município de São Luís, enquanto que, para o município de São Bento, obteve-se o primeiro relato para a família Strigeidae.

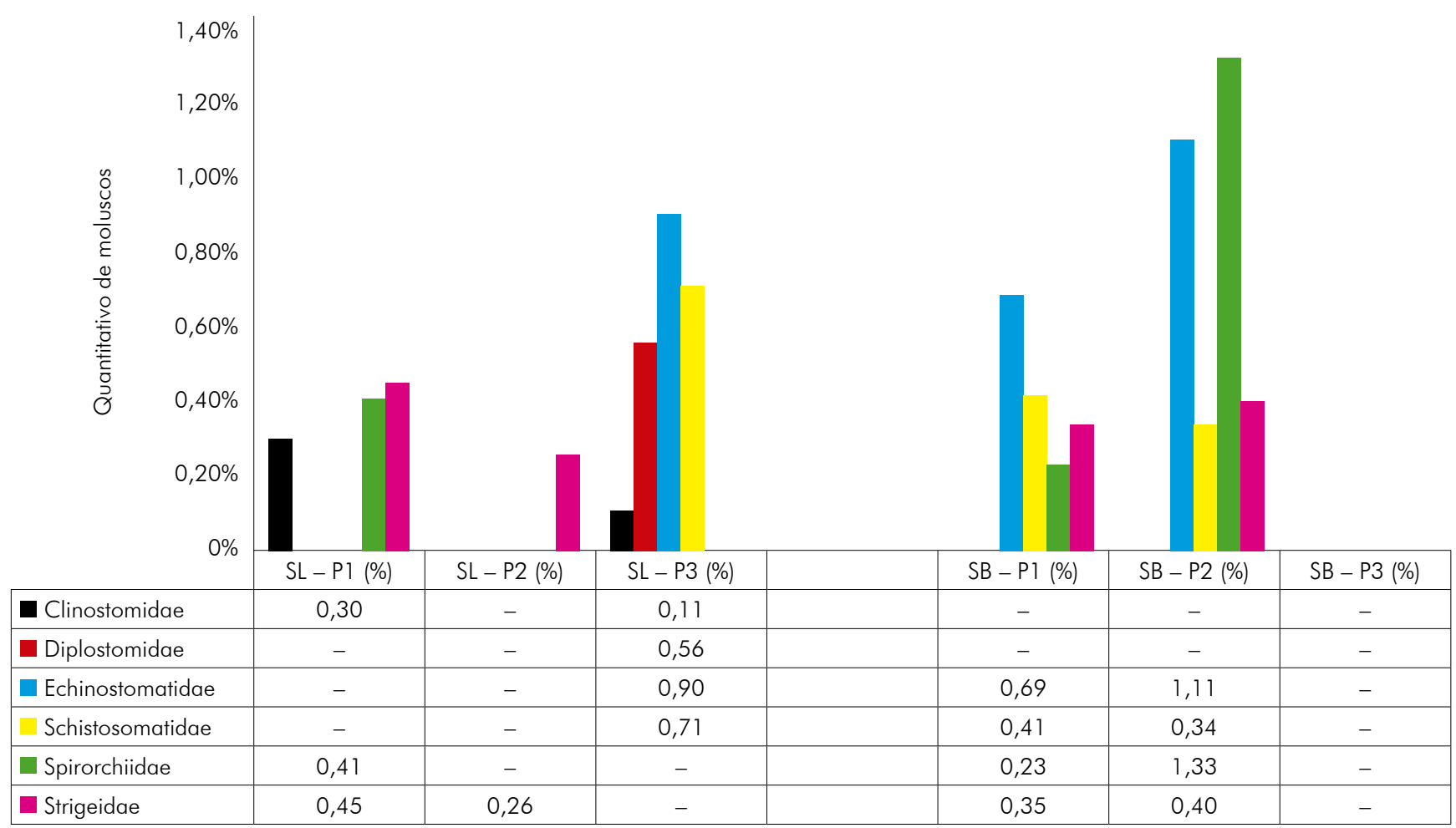

SL: São Luís; SB: São Bento; Sinal convencional utilizado: - Dado numérico igual a zero, não resultante de arredondamento.

Figura 4 - Percentual de moluscos positivos para seis famílias de larvas de trematódeos encontradas nos municípios de São Luís e São Bento, estado do Maranhão, Brasil, durante o período de fevereiro de 2015 a janeiro de 2016 
Tabela 1 - Percentual de diferentes larvas de trematódeos encontrados em exemplares de Biomphalaria spp. coletados nos municípios de São Luís e São Bento, estado do Maranhão, Brasil, entre fevereiro de 2015 e janeiro de 2016

\begin{tabular}{lccc}
\hline $\begin{array}{c}\text { Larvas de } \\
\text { trematódeos }\end{array}$ & Local & Molusco & $\begin{array}{c}\text { Percentual } \\
\text { de infeç̧ão }\end{array}$ \\
\hline Clinostomidae & São Luís & B. straminea & $0,41 \%(11 / 2.661)$ \\
Diplostomidae & São Luís & B. straminea & $0,56 \%(15 / 2.661)$ \\
Echinostomatidae & São Luís & B. glabrata & $0,90 \%(24 / 2.661)$ \\
& São Bento & B. glabrata & $1,80 \%(31 / 1.726)$ \\
Schistosomatidae & São Luís & B. glabrata & $0,71 \%(19 / 2.661)$ \\
& São Bento & B. glabrata & $0,75 \%(13 / 1.726)$ \\
& São Luís & B. straminea & $0,26 \%(7 / 2.661)$ \\
Spirorchiidae & S. glabrata & $0,15 \%(4 / 2.661)$ \\
& São Bento & B. straminea & $0,40 \%(7 / 1.726)$ \\
& B. glabrata & $1,16 \%(20 / 1.726)$ \\
Strigeidae & São Luís & B. straminea & $0,71 \%(19 / 2.661)$ \\
& & B. glabrata & $0,75 \%(13 / 1.726)$ \\
\hline & & &
\end{tabular}

\section{DISCUSSÃO}

Os pontos que apresentaram maior quantidade de caramujos coletados e maior índice de positividade para algum tipo de cercária, tanto em São Luís quanto em São Bento, foram os pontos (P3 e P2, respectivamente) localizados próximos às residências, sendo encontrados caramujos até mesmo em valas e córregos das calçadas de concreto (criadouro artificial). A elevada abundância de caramujos nesses locais pode estar correlacionada com sua preferência por habitat antropogênicos, ricos em matéria orgânica ${ }^{37,38}$. Em relação aos criadouros que apresentaram baixa abundância de caramujos, pode ser devido a fatores como a presença de substrato sujeito a modificações - por exemplo, a ocorrência de forte correnteza, evidenciada em P2 (São Luís) e P3 (São Bento) - possuindo, dessa forma, baixa disponibilidade de matéria orgânica, o que provavelmente estaria interferindo na oferta de alimento e, consequentemente, em uma menor colonização do criadouro $^{39}$.

Em São Luís, houve maior percentual de moluscos eliminando cercárias das famílias Echinostomatidae $(0,90 \%)$, Strigeidae $(0,71 \%)$ e Schistosomatidae $(0,71 \%)$, sendo essa última representada somente por cercárias causadoras da doença esquistossomose mansônica. Essas cercárias foram encontradas somente em P3, que ficava localizado em um bairro periférico da zona urbana de São Luís. Nesse mesmo local, Oliveira et al. ${ }^{40}$ realizaram um inquérito malacológico e detectaram seis planorbídeos contaminados pelo parasito, relatando a presença dessa parasitose na área.

Em P3 de São Luís, constatou-se uma maior biodiversidade de larvas, sendo encontrados quatro tipos distintos. Segundo Hudson et al. ${ }^{41}$ e Minguez et al. ${ }^{42}$, essa elevada diversidade e abundância de trematódeos em moluscos pode estar relacionada à presença de vários outros táxons de vertebrados necessários para a manutenção dos respectivos ciclos biológicos heteróxenos de diversos parasitos, indicando uma condição ambiental favorável em relação ao funcionamento trófico. Tal condição ambiental foi observada no referido ponto de coleta, o que provavelmente confirma essa hipótese.

Em São Bento, houve maior percentual de caramujos eliminando cercárias das famílias Echinostomatidae $(1,80 \%)$ e Spirorchiidae $(1,56 \%)$. Para O P2 desse município, verificou-se uma maior quantidade de eliminação dessas cercárias pelos moluscos. Desses planorbídeos, $\quad 0,75 \% \quad(13 / 1.726)$ encontravam-se positivos para S. mansoni (Schistosomatidae), notificando também a presença da esquistossomose mansônica nessa localidade. Para esse município, Cantanhede et al. ${ }^{21}$ verificaram a biodiversidade de moluscos e de larvas de trematódeos, encontrando 0,22\% (1/444) de B. glabrata eliminando cercárias de S. mansoni. Confirmou-se, assim como em São Luís, a presença desse parasito.

As taxas de eliminação de cercárias de S. mansoni por moluscos Biomphalaria spp., verificadas nesta pesquisa para P1 e P2 de São Bento, considerada uma área endêmica para a esquistossomose ${ }^{24,43}$, não foram elevadas quando comparadas às taxas de eliminação dessas mesmas cercárias pelos moluscos do P3 em São Luís. Isso pode estar relacionado com a elevada presença de larvas da família Echinostomatidae, eliminadas por moluscos desses pontos em São Bento, pois, segundo alguns autores ${ }^{44,45,46,47,48}$, cercárias dessa família podem interferir negativamente no ciclo biológico das formas larvais de S. mansoni, provocando alterações fisiológicas nos moluscos vetores que, possivelmente, impedem a instalação e evolução do miracídio, possuindo, assim, uma relação de antagonismo direto e/ou indireto com o mesmo.

Todavia, como a doença é constatada desde 1920 no município de São Bento, segundo Alvim ${ }^{43}$, a baixa ocorrência do parasito pode estar relacionada com a maioria da população vivendo com baixos níveis de parasitismo, devido a sucessivas reinfecções, não apresentando, assim, quantidade suficiente de ovos eliminados nas fezes, o que poderia diminuir a probabilidade de infecção dos caramujos.

Nos estudos de Souza et al. ${ }^{16}$ e Pinto e Melo ${ }^{5}$, as cercárias Strigeidae, Clinostomidae, Echinostomatidae e Spirorchiidae foram eliminadas de até três espécies de Biomphalaria (B. glabrata, B. straminea e Biomphalaria tenagophila). Já nos estudos de Silva e Melo49, Strigeidae e Diplostomidae foram eliminadas de $B$. glabrata e B. tenagophila. Em outros levantamentos e estudos, Schistosomatidae, Strigeidae e Diplostomatidae 
foram eliminadas somente de B. glabrata $^{13,50}$; e Echinostomatidae e Strigeidae, de B. tenagophila ${ }^{17,51}$.

\section{CONCLUSÃO}

A porção oriental da Amazônia Legal brasileira, representada neste estudo pelos municípios maranhenses, São Bento e São Luís, apresentou elevada biodiversidade de larvas de trematódeos, sendo que os criadouros naturais que continham maior quantidade de caramujos infectados foram os mais próximos das residências. Não houve diferenças na diversidade das famílias de trematódeos eliminadas entre as duas espécies de Biomphalaria identificadas na região. Nesta pesquisa, foram relatados os primeiros registros de diferentes cercárias para ambos os municípios. Desse modo, maiores esforços para ampliação do conhecimento sobre essas formas evolutivas de trematódeos digenéticos na Região Amazônica devem ser realizados.

\section{AGRADECIMENTOS}

À UEMA, pelo transporte para as coletas, sendo essencial para a execução desta pesquisa.

\section{APOIO FINANCEIRO}

UEMA, por meio de bolsa de iniciação científica ao primeiro autor.

\section{REFERÊNCIAS}

1 Combes C, Fournier A, Moné $H$, Théron A. Behaviours in trematode cercariae that enhance parasite transmission: patterns and processes. Parasitology. 1994 Jan;109 Suppl:S3-13.

2 Sukhdeo MVK, Sukhdeo SC. Trematode behaviours and the perceptual worlds of parasites. Can J Zool. 2004 Feb;82(2):292-315.

3 Koehler AV, Brown B, Poulin R, Thieltges DW, Fredensborg BL. Disentangling phylogenetic constraints from selective forces in the evolution of trematode transmission stages. Evol Ecol. 2012 Nov;26(6): 1497-512.

4 Morley NJ. Cercariae (Platyhelminthes: Trematoda) as neglected components of zooplankton communities in freshwater habitats. Hydrobiologia. 2012 Jul;691(1):7-19.

5 Pinto HA, Melo AL. Larvas de trematódeos em moluscos do Brasil: panorama de perspectivas após um século de estudos. Rev Patol Trop. 2013 out-dez;42(4):387-94.

6 Esch GW, Barger MA, Fellis KJ. The transmission of digenetic trematodes: style, elegance, complexity. Integr Comp Biol. 2002 Apr;42(2):304-12.

7 Lutz A. Introdução ao estudo da evolução dos endotrematodes brasileiros. Mem Inst Oswaldo Cruz. 1922;14(1):95-103

8 Ruiz JM. Contribuição ao estudo das formas larvárias de trematódeos brasileiros. An Fac Farm Odontol Univ Sao Paulo. 1943;3:105-12.

9 Ruiz JM. Contribuição ao estudo das formas larvárias de trematódeos brasileiros. 2. Fauna de Santos, Estado de São Paulo. Mem Inst Butantan. 1952;24:17-36.

10 Ruiz JM. Contribuição ao estudo das formas larvárias de trematódeos brasileiros. 3. Fauna de Belo Horizonte e Jaboticatubas. Estado de Minas Gerais. Mem Inst Butantan. 1952;24:45-62.
11 Ruiz JM. Contribuição ao estudo das formas larvárias de trematódeos brasileiros. 5. Descrição das três furcocercárias que ocorrem em planorbídeos hospedeiros do Schistosoma mansoni. Mem Inst Butantan. 1953;25:77-89.

12 Boaventura MF, Thiengo SC, Fernandez MA. Gastrópodes límnicos hospedeiros intermediários de trematódeos digenéticos no Brasil. In: Santos SB, Pimenta AD, Thiengo SC, Fernandez MA, Absalão RS, editores. Tópicos em Malacologia: ecos do XVIII Encontro Brasileiro de Macologia. Rio de Janeiro: Sociedade Brasileira de Malacologia; 2007. p. 327-37.

13 Souza MAA, Melo AL. Caracterização de larvas de trematódeos emergentes de moluscos gastrópodes coletados em Mariana, Minas Gerais, Brasil. Iheringia Ser Zool. 2012 mar;102(1):11-8.

14 Veitenheimer-Mendes IL. Cercárias em Biomphalaria tenagophila (Orbigny, 1835) (Mollusca, Planorbidae) de Guaíba, Rio Grande do Sul, Brasil. Iheringia Ser Zool. $1981 ;(60): 3-12$.

15 Espíndola KS, Machado MM, Hofmann PRP. Natural and experimental infection of planorbids from the island of Santa Catarina (Brazil). Rev Inst Med Trop Sao Paulo. 1992 Jul-Aug;34(4):289-94.

16 Souza CP, Lima LC, Jannotti-Passos LK, Ferreira SS, Guimarães CT, Vieira IBF, et al. Moluscos límnicos da microrregião de Belo Horizonte, MG, com ênfase nos vetores de parasitoses. Rev Soc Bras Med Trop. 1998 set-out;31 (5):449-56.

17 Moraes J, Silva MPN, Ohlweiler FP, Kawano T. Schistosoma mansoni and other larval trematodes in Biomphalaria tenagophila (Planorbidae) from Guarulhos, São Paulo State, Brazil. Rev Inst Med Trop Sao Paulo. 2009 Mar-Apr;51 (2):77-82.

18 Steinmann P, Keiser J, Bos R, Tanner M, Utzinger J. Schistosomiasis and water resources development: systematic review, meta-analysis, and estimates of people at risk. Lancet Infect Dis. 2006 Jul;6(2):411-25. 
19 Keiser J, Utzinger J. Food-borne trematodiases. Clin Microbiol Rev. 2009 Jul;22(3):466-83.

20 Soldánová M, Selbach C, Kalbe M, Kostadinova A, Sures B. Swimmer's itch: etiology, impact, and risk factors in Europe. Trends Parasitol. 2013 Feb;29(2):65-74.

21 Cantanhede SPD, Fernandez MA, Mattos AC, Montresor LC, Silva-Souza N, Thiengo SC. Freshwater gastropods of the Baixada Maranhense Microregion, an endemic area for schistosomiasis in the State of Maranhão, Brazil: I - qualitative study. Rev Soc Bras Med Trop. 2014 Jan-Feb; 47(1):79-85.

22 Rocha GM. Aspectos recentes do crescimento e distribuição da população da Amazônia Brasileira. In: Aragón LE, organizador. Populações da Pan-Amazônia. Belém: NAEA; 2005. p. 141-9.

23 Procópio A. Destino amazônico. São Paulo: Hucitec; 2006.

24 Silva-Souza N, Vasconcelos SD. Histopathology of Holochilus brasiliensis (Rodentia: Cricetidae) infected with Schistosoma mansoni (Schistosomatida: Schistosomatidae). Rev Patol Trop. 2005 MayAug;34(2): 145-50.

25 Costa-Neto JP, Barbieri R, Ibañez MSR, Cavalcante PRS, Piorski NM. Limnologia de três ecossistemas aquáticos característicos da Baixada Maranhense. Bol Lab Hidrob. 2002;14-15(1):19-38.

26 Secretaria de Estado do Meio Ambiente e Recursos Hídricos (Maranhão). Diagnóstico ambiental da microrregião da aglomeração urbana de São Luís e dos Municípios de Alcântara, Bacabeira e Rosário. São Luís: SEMA; 1988.

27 Instituto Brasileiro de Geografia e Estatística. Cidades@: Maranhão [Internet]. Rio de Janeiro: IBGE; 2010 [citado 2016 mar 22]. Disponível em: http://cidades.ibge.gov.br/painel/painel.php?lan $\mathrm{g}=\& \operatorname{codmun}=211130 \&$ search $=$ maranhao $\mid$ saoluis |infograficos:-dados-gerais-do-municipio.

28 Malek EA. Snail hosts of schistosomiasis and other snail-transmitted diseases in tropical America: a manual. Washington: PAHO; 1985. p. 300-10. (PAHO. Scientific públication; 478).

29 Paraense WL, Deslandes N. Observations on the morphology of Australorbis nigricans. Mem Inst Oswaldo Cruz. 1955 May;53(1):121-4.

30 Paraense WL. Estado atual da sistemática dos planorbídeos brasileiros. Arq Mus Nac. 1975;55:105-28.

31 Ministério da Saúde (BR). Secretaria de Vigilância em Saúde. Vigilância e controle de moluscos de importância epidemiológica. Diretrizes técnicas: Programa de Vigilância e Controle da Esquistossomose (PCE). 2. ed. Brasília: Ministério da Saúde; 2008.
32 Schell SC. How to know the trematodes. Dubuque: William C. Brown Company Publishers; 1970.

33 Combes C, Albaret JL, Arvy L, Bartoli P, BayssadeDufour C, Deblock S, et al. Atlas mondial des cercaires. Paris: Éditions du Muséum National d'Histoire Naturelle; 1980. (Nouvelle série, série A, Zoologie; 115).

34 Gibson DI, Jones A, Bray RA, editors. Keys to the Trematoda. Vol. 1. London: CABI publishing, Wallingford, UK and the Natural History Museum; 2002.

35 Jones A, Bray R, Gibson DI, editors. Keys to the Trematoda. Vol. 2. London: CABI publishing, Wallingford, UK and the Natural History Museum; 2005.

36 Bray RA, Gibson DI, Jones A, editors. Keys to the Trematoda. Vol. 3. London: CABI publishing, Wallingford, UK and the Natural History Museum; 2008.

37 Bini LM, Thomaz SM, Murphy KJ, Camargo AFM. Aquatic macrophyte distribution in relation to water and sediment conditions in the Itaipu Reservoir, Brazil. Hydrobiologia. 1999 Nov;4 15:147-54.

38 Abílio FJP, Fonseca-Gessner AA, Leite RL, Ruffo TLM. Gastrópodes e outros invertebrados do sedimento e associados à macrófita Eichhornia crassipes de um açude hipertrófico do semiárido paraibano. Rev Biol Cienc Terra. 2006;1 supl: 165-78.

39 Janasi VA, Negri FA, Montanheiro TJ, Freitas VA, Rocha BC, Reis PM. Geochemistry of the eocretacic basalt magmatism in the Piraju-Ourinhos region, SE Brazil, and implications to the stratigraphy of the Serra Geral Formation. Rev Bras Geocienc. 2007 Mar;37(1):148-62.

40 Oliveira DS, Nunes GS, Mendes RJ, França CRC, Pereira Filho AA, Tavares $C P$, et al. Inquérito malacológico para identificar a célula de expansão da esquistossomose mansônica na Vila Embratel, um bairro de periferia de São Luís do Maranhão. Cad Pesq. 2013 jul;20(no. esp):16-9.

41 Hudson PJ, Dobson AP, Lafferty KD. Is a healthy ecosystem one that is rich in parasites? Trends Ecol Evol. 2006 Jul;21 (7):381-5.

42 Minguez L, Molloy DP, Guérold F, Giambérini L. Zebra mussel (Dreissena polymorpha) parasites: potentially useful bioindicators of freshwater quality? Water Res. 2011 Jan;45(2):665-73.

43 Alvim MCA. Esquistossomose mansoni no Maranhão. Hileia Med. 1980;2(2):151-7.

44 Lim HK, Heyneman D. Intramolluscan inter-trematode antagonism: a review of factors influencing the hostparasite system and its possible role in biological control. Adv Parasitol. 1972 Jan;10:191-268. 
45 Kuris AM. Biological control: implications of the analogy between the trophic interactions of insect pest-parasitoid and snail-trematode systems. Exp Parasitol. 1973 Apr;33(2):365-79.

46 Combes C. Trematodes: antagonism between species and sterilizing effects on snails in biological control. Parasitology. 1982 Apr;84(4):151-75.

47 Machado SMP, Magalhães LA, Artigas PT, Cordeiro NS, Carvalho JF. Verificação de antagonismo entre larvas de Schistosoma mansoni e larvas de outros Digenea em Biomphalaria tenagophila, molusco planorbídeo de criadouro natural situado na região de Campinas, SP, Brasil. Rev Saude Publica. 1988 dez;22(6):484-8.

48 Esteban JG, Muñoz-Antoli C, Trelis M, Toledo R. Effects of nonschistosome larval trematodes on Biomphalaria snails. In: Toledo R, Fried B, editors. Biomphalaria snails and larval trematodes. New York: Springer; 2011 . p. 127-58.
49 Silva RE, Melo AL. Caracterização de larvas de trematódeos emergentes de moluscos de água doce coletados na bacia hidrográfica do Lago Soledade, Ouro Branco, Minas Gerais, Brasil. Lundiana. 2013;11(1-2):21-33.

50 Souza MAA, Barbosa VS, Wanderlei TNG, Barbosa CS. Criadouros de Biomphalaria, temporários e permanentes, em Jaboatão dos Guararapes, PE. Rev Soc Bras Med Trop. 2008 mai-jun;4 1 (3):252-6.

51 Boaventura MF, Fernandez MA, Thiengo SC, Silva RE, Melo AL. Formas larvais de Trematoda provenientes de gastrópodes límnicos da microrregião Rio de Janeiro, sudeste do Brasil. Lundiana. 2002;3(1):45-9. 